\title{
The Effect of Porogen Loading on the Stiffness and Fracture Energy of Brittle Organosilicates
}

\section{Citation}

Li, Han, Youbo Lin, Ting Y. Tsui, and Joost J. Vlassak. 2009. The effect of porogen loading on the stiffness and fracture energy of brittle organosilicates. Journal of Materials Research 24(1): 107-116.

\section{Published Version}

doi:10.1557/jmr.2009.0005

\section{Permanent link}

http://nrs.harvard.edu/urn-3:HUL.InstRepos:4263767

\section{Terms of Use}

This article was downloaded from Harvard University's DASH repository, and is made available under the terms and conditions applicable to Open Access Policy Articles, as set forth at http:// nrs.harvard.edu/urn-3:HUL.InstRepos:dash.current.terms-of-use\#OAP

\section{Share Your Story}

The Harvard community has made this article openly available.

Please share how this access benefits you. Submit a story.

\section{Accessibility}




\title{
The effect of porogen loading on the stiffness and fracture energy of brittle organosilicates
}

\author{
Han Li, ${ }^{a}$ Youbo Lin, ${ }^{a}$ Ting Y. Tsui, ${ }^{b}$ Joost J. Vlassak ${ }^{a,}{ }^{*}$ \\ ${ }^{a}$ School of Engineering and Applied Sciences, Harvard University, \\ Cambridge, MA 02138 \\ ${ }^{\mathrm{b}}$ Chemical Engineering Department, University of Waterloo, Nanotechnology Institute \\ Waterloo, Ontario N2L 3G1, Canada \\ ${ }^{*}$ Corresponding author. Tel.: +1-617-496-0424; fax: +1-617-496-0601. \\ E-mail address: vlassak@esag.deas.harvard.edu (J.J. Vlassak).
}

\begin{abstract}
$\underline{\text { Abstract }}$
Integrating porous low-permittivity dielectrics into $\mathrm{Cu}$ metallization is one of the strategies to reduce power consumption, signal propagation delays, and crosstalk between interconnects for the next generation of integrated circuits. The porosity and pore structure of these low-k dielectric materials, however, also affect other important material properties in addition to the dielectric constant. In this paper, we investigate the impact of porogen loading on the stiffness and cohesive fracture energy of a series of porous organosilicate glass (OSG) thin films using nanoindentation and the doublecantilever beam (DCB) technique. The OSG films were deposited by plasma-enhanced chemical vapor deposition (PECVD) and had a porosity in the range of $7 \sim 45 \%$. We show that the degree of porogen loading during the deposition process changes both the network structure and the porosity of the dielectric, and we resolve the contributions of both effects to the stiffness and fracture energy of the films. The experimental results for stiffness are compared with micromechanical models and finite element calculations.
\end{abstract}


It is demonstrated that the stiffness of the OSG films depends sensitively on their porosity and that considerable improvements in stiffness may be obtained through further optimization of the pore microstructure. The cohesive fracture energy of the films decreases linearly with increasing porosity, consistent with a simple planar through-pore fracture mechanism.

\section{Introduction}

Nanoporous OSG coatings with relative dielectric constants smaller than 2.5 are considered for use as intra-metal dielectric in future generations of advanced integrated circuits. Their low dielectric constant is achieved by introducing a substantial fraction of nanometer-sized pores into a hybrid organic-inorganic matrix [1-3]. Subtractive porosity levels of up to $45 \%$ can be reached by changing the loading of porogen (pore generator) during the film deposition process. While introducing porosity may be beneficial for the dielectric properties of the coatings, porosity does have a negative impact on the mechanical properties of the coatings, which are critical to the integrity of the dielectrics during integrated circuit fabrication [1-3].

Nemat-Nasser and Hori reviewed a large body of theoretical work on modeling the effect of porosity on the bulk elastic response of porous materials [4]. Micromechanics models [5-7], which combine the exact Eshelby inclusion solution [8] and various assumptions on approximating the deformation interaction between pores, can capture the effect of porosity and pore shape on the effective elastic constants explicitly, but are 
generally limited to structures with simple pore geometries. Semi-empirical formulae can be derived from the minimum solid area model [9]. The microstructure that corresponds to this model is, however, not clearly defined limiting the predictive and interpretative power of this method. Torquato et al. describe two-phase media (including random porous structures) using an n-point probability function from which an exact or approximate effective modulus tensor can be derived depending on the order of the series expansion [10]. The difficulty of this approach lies in the limited knowledge one has of the probability functions for a given porous material. Roberts et al. evaluate the effective Young's modulus and Poisson's ratio for several ideal structures, including the random overlapping solid sphere model and the random overlapping spherical pore model using finite element calculations [11, 12]. The effects of porosity and pore geometry can be studied separately and clear structure-property correlations are readily seen. In principle, this methodology can also be applied to other structures.

With regards to the fracture behavior of porous materials, Vandeperre et al. [13] studied the effect of porosity on the fracture energy of bulk porous alumina and poly(methyl methacrylate) (PMMA), a brittle and transparent polymer, using the single edge notch beam (SENB) test. Their results showed that the fracture energy was largely constant when the level of porosity was below a certain critical value (about 15 25\% for their alumina samples); at larger porosity levels, the fracture energy decreased rapidly with increasing porosity. Direct observation of crack propagation in PMMA with drilled cylindrical pores revealed that cracks did not cut straight through the pores, but that the crack front was curved in the vicinity of a pore. No detectable effect of pore 
size was observed as long as the pores were sufficiently small. Guyer et al. studied the cohesive fracture properties of low-k methylsilsesquioxane (MSSQ) thin films of different mass densities, and hence different overall porosities, using the four-point bend method [14]. The cohesive fracture energy of the MSSQ films was shown to scale with average film density (porosity) through an empirical power-law relation with an exponent equal to 2 . The authors attributed the nonlinear scaling behavior in part to contact friction between the crack faces under the mixed loading condition of the fourpoint bending test and to the non-uniform density of the MSSQ through the coating thickness. It is well known that cracks in the four-point bend test tend to propagate at or near the top interface of the film as a result of the mixed loading conditions (phase angle $\sim 42^{\circ}$ ). Since the local properties at an interface can be quite different from those in the bulk of the film[15], the results obtained by Guyer et al. using four point bending are not necessarily representative of the bulk film properties. Hence, it is of interest to investigate the cohesive fracture energy under pure mode I conditions, where the fracture path typically lies within the bulk of the film and where friction between crack faces is minimized.

In this paper, we first present experimental measurements of the stiffness and cohesive fracture energy of a series of OSG films deposited with different levels of porogen content. In addition to changing the final porosity of the films in the range of $7 \%$ to $45 \%$, the porogen also alters the network structure and hence the properties of the dielectric matrix. We evaluate the contributions of both the porosity and the altered network structure on the stiffness and the fracture energy of the films. We then discuss finite element simulations performed to determine the effect of the pore microstructure 
on the elastic response of the OSG and we demonstrate that substantial improvement in stiffness may be achieved by further optimizing the pore microstructure. In the last section we discuss the cohesive fracture energy of the films and show that the fracture energy decreases linearly with increasing porosity, consistent with a simple planar through-pore fracture mechanism.

\section{$\underline{\text { Experimental }}$}

Porous OSG films were deposited at $250{ }^{\circ} \mathrm{C}$ onto $300-\mathrm{mm}$ (100)-Si wafers by means of plasma-enhanced chemical vapor deposition (PECVD) using diethoxymethylsilane (DEMS) as a precursor, with helium and oxygen as carrier gas and oxidizer, respectively. A proprietary aromatic porogen was added to the gas mix to form the sacrificial porogen phase. Pores were formed in the films after the deposition process by removing this porogen phase from the as-deposited films. This operation was performed by irradiating the as-deposited films at $400{ }^{\circ} \mathrm{C}$ with white UV radiation in vacuum for a period of time that depended on film thickness. The precise duration of the irradiation process was determined so as to minimize the dielectric constant while enhancing the mechanical strength of the resulting coating. At short irradiation times, only part of the porogen was broken down and vaporized from the matrix, and the dielectric constant and density of the coating decreased with further irradiation. At long times, all of the porogen was removed, but the UV radiation densified the OSG matrix, resulting in a slow rise of dielectric constant and the strength of the film. As a guide, it took approximately $10 \mathrm{~min}$ to remove the porogen phase and to obtain sufficient strength for a $280 \mathrm{~nm}$-thick film with a porosity of $27.2 \%$. The level of porosity in each

film was controlled by changing the porogen loading during the deposition process. The 
highest level of porosity that was reached using this approach was $45 \%$. Without any porogen loading, the OSG had a porosity of $7 \%$ and this value represents the lower end of the porosity spectrum studied in this paper. The relative dielectric constant of the coatings was measured at a frequency of $1 \mathrm{MHz}$ using a KLA Quantox XP metrology system, and the results are listed in Table I. We measured the film porosity with ellipsometry-based porosimetry [16] using a Woollam VASE spectrometer with toluene as the probe species. The reported values of porosity (Table I) are the average of values obtained from the adsorption and desorption isotherms. The precise pore size is not known, but it is expected to be on the order of $2 \mathrm{~nm}$ based on reports in the literature for similar coatings [1].

The structure of the OSG films was characterized by FTIR spectroscopy in transmission mode using a Nicolet NEXUS 670 spectrometer equipped with an MCT-A detector and a $\mathrm{KBr}$ beam splitter. The infrared beam was incident on the backside of the polished Si substrate at an angle of $47^{\circ}$ to the surface normal. All spectra were collected at a resolution of $2 \mathrm{~cm}^{-1}$ in a nitrogen-purged cell; a common background spectrum obtained under the same condition using a bare silicon substrate was subtracted from all spectra. The transmittance of the specimens was recorded from 4000 to $500 \mathrm{~cm}^{-1}$, and the absorbance was determined from the transmittance using $\alpha=-\log _{10} T$, where $\alpha$ is the absorbance and $T$ is the transmittance. All spectra were normalized by film thickness according to the Beer-Lambert law and Gaussian multi-peak fitting was used to analyze overlapping absorption bands with the peak position, amplitude and width of each Gaussian as free variables. The absorption spectrum provides information on the functional groups that are present in the films. The infrared absorption inverse cross- 
sections of the various functional groups reported in reference $[17,18]$ were used to calculate the network bond densities of Si-C and Si-O bonds in the OSG films.

The elastic moduli of the OSG films were measured using a Nanoindenter XP system (MTS System Corporation, Oak Ridge, TN) equipped with a diamond Berkovich tip (i.e., a three-sided pyramidal tip). In this technique, the elastic moduli of the films were determined from the contact stiffness between the films and the indenter tip using the analysis developed by Oliver and Pharr [19]. The indentation experiments were conducted in the continuous stiffness measurement mode, in which a small oscillation at a frequency of $40 \mathrm{~Hz}$ is imposed on the indentation force, so that the plane-strain modulus, $M=E /\left(1-v^{2}\right)$, of the film can be measured continuously as a function of indentation depth according to

$$
S=2 \beta \cdot M_{r} \sqrt{A / \pi},
$$

where $S$ is the experimental contact stiffness, $\beta$ is a tip geometry constant $(\beta=1.034$ for a Berkovich tip), $A$ is the projected contact area between indenter tip and film, and $M_{r}=\left(M^{-1}+M_{\text {diamond }}^{-1}\right)^{-1}$ is the reduced indentation modulus. The plane-strain modulus of the films is reported instead of Young's modulus $E$ because Poisson's ratio of the films is difficult to measure accurately and is generally not known. One concern when measuring the stiffness of compliant films on substrates is the contribution of the substrate to the experimental contact stiffness. This contribution depends on the elastic mismatch between film and substrate and also on the ratio of indentation depth over film thickness [20]. To minimize the effect of the substrate on the experimental film stiffness, nanoindentation experiments were performed on OSG films with a thickness 
of $2300 \mathrm{~nm}$. A total of ten indentations experiments were conducted and averaged per film. As is evident in Fig. 1, the plane-strain moduli of the films were constant for indentation depths in the range from 50 to $200 \mathrm{~nm}$, i.e., for depths less than $10 \%$ of the film thickness, as expected based on FEM indentation simulations for compliant films on stiff substrates [20]. The indentation moduli were taken as the averages in the plateau regions and experimental values are reported in Table I. The errors reported in the table correspond to the standard deviations of the moduli for indentation depths ranging from 50 to $200 \mathrm{~nm}$.

The films for the fracture measurements were deposited under the same condition as those for the stiffness measurements, but with a thickness of $280 \mathrm{~nm}$. After deposition, the films were capped with a $90 \mathrm{~nm}$ PECVD SiN $\mathrm{x}_{\mathrm{x}}$ coating. The cohesive fracture energy of the OSG films was measured using the double-cantilever beam (DCB) method, which provides a mode I loading configuration confining the crack within the OSG films, rather than along the interface. The DCB specimens were fabricated using the following procedure. First the film stacks were sandwiched between two pieces of silicon using a $5 \mu \mathrm{m}$ thick spin-on epoxy (Epotek_353ND). Prior to spin-coating the epoxy, a $40 \mathrm{~nm}$ Ti adhesion layer and a $400 \mathrm{~nm} \mathrm{Cu}$ layer were sputter deposited onto the OSG film stack to increase the distance between the OSG film and the epoxy and thus to minimize any energy dissipation in the epoxy during the measurement. The epoxy was cured at $120{ }^{\circ} \mathrm{C}$ for $1 \mathrm{~h}$ and the resulting sandwich was diced into six specimens with dimensions $60 \mathrm{~mm} \times 10 \mathrm{~mm}$ and with the long edge parallel to the $<110>$ direction of the Si wafer, using a DISCO ADA-321 high-speed saw. Pre-cracks 
were created at one end of the DCB specimens by driving a razor blade between the two silicon pieces until a crack length of $10 \mathrm{~mm}$ was reached.

All DCB experiments were performed under dry flowing $\mathrm{N}_{2}$ to keep the relative humidity below $10 \%$. The specimens were loaded by separating the ends of the specimen arms at a constant speed of $30 \mu \mathrm{m} / \mathrm{s}$, resulting in a crack velocity on the order of $25 \mu \mathrm{m} / \mathrm{s}$. At least five specimens were tested for each set of experimental conditions. The energy release rate to drive the crack in the OSG under these conditions was taken to be the cohesive fracture energy of the OSG. The energy release rate in the doublecantilever beam configuration is given by $[21,22]$

$$
G=\frac{12\left(1-v^{2}\right) P^{2} a^{2}}{E t^{3} b^{2}}\left(1+0.64 \frac{t}{a}\right)^{2},
$$

where $a$ is the crack length, $P$ is the applied force, $E$ and $v$ are Young's modulus and Poisson's ratio of the substrate, $b$ is the width of the sample, and $t$ is the thickness of the substrate. The crack length $a$ can be evaluated by measuring the sample compliance [21, 22]

$$
a=\left(\frac{E b h t^{3}}{8 P\left(1-v^{2}\right)}\right)^{1 / 3}-0.64 t
$$

where $h$ the crack opening distance as measured at the open end of the sample. Young's modulus and Poisson's ratio of the silicon substrate were taken as $168.9 \mathrm{GPa}$ and 0.064 , respectively, as appropriate for the crystallographic orientation of the samples. After the fracture test, the crack faces were analyzed by x-ray photoelectron spectroscopy (XPS) using an SSX-100 XPS system to determine the location of the crack path. In all cases, the fracture path lay within the OSG film. Figure 2 shows representative XPS spectra 
for an OSG film with a porosity of $14.8 \%$. The spectra for both crack faces are typical for OSG and there is no visible nitrogen peak as one would expect if the crack propagated at the $\mathrm{OSG} / \mathrm{SiN}_{\mathrm{x}}$ interface. The morphology of the fracture surface was examined at randomly selected positions near the center of the fracture surface by means of atomic force microscopy (AFM). These measurements were performed in tapping mode using an Asylum MFP-3D ${ }^{\text {TM }}$ Stand Alone system equipped with an ultrasharp tip (nominal tip radius of curvature $<15 \mathrm{~nm}$ ). The AFM scan area (from $0.5 \mu \mathrm{m}$ to $50 \mu \mathrm{m}$ on a side) and step size (from $0.5 \mathrm{~nm}$ to $5 \mathrm{~nm}$ ) were varied systematically to examine the roughness of the crack faces. The AFM images were flattened by a first order plane correction to eliminate tilting and analyzed using the MFP-3D ${ }^{\mathrm{TM}}$ software by Asylum Research.

\section{Finite element modeling}

Roberts et al. have used the finite element method (FEM) to model the relationship between the level of porosity of a solid and its elastic properties for a number of representative microstructures, including the random overlapping spherical pore model [11], the random overlapping sphere model [11], and the open cell model [23]. We have reproduced the results obtained by Roberts et al. for the overlapping sphere microstructure and have applied the same methodology to analyze a microstructure that consists of random non-overlapping spherical pores, which has not yet been explored to our knowledge. The motivation for analyzing this particular microstructure lies in the fact that the isolated closed-pore microstructure is desirable for a porous low-k dielectric because this microstructure minimizes the ingress of chemical species such as water or various precursors that adversely affect the dielectric [2, 3]. 
Three-dimensional finite element models containing non-overlapping spherical pores were created by cutting spherical pores, one at a time, at randomly selected position within a solid cube. New pores were only allowed if they did not overlap with other pores. This process of pore generation was repeated until a specified porosity was reached. Using this method, microstructures with porosity levels in the range of $0-40 \%$ were explored. All FEM calculations were performed using the commercial code ABAQUS with 10-node quadratic tetrahedral elements and with a refined mesh in the vicinity of the pores. In most calculations, the pore diameter was fixed at $1 \%$ of the edge length of the model. The effect of pore size distribution on the stiffness was evaluated by comparing two distinct microstructures with nominally the same level of porosity: one microstructure with a uniform pore size and one in which the pore diameter was uniformly distributed in the range of 0.5 to $1.5 \%$ of the edge length of the model.

The effective stiffness of the porous structure was calculated by imposing a uniform normal displacement on two opposite faces of the cube, while enforcing traction-free boundary conditions on the other faces. The loaded faces were allowed to contract or expand laterally without any constraint. The average normal stress was calculated from the nodal reaction forces on one of the loaded faces and the nominal cross-sectional area. At least five random structures were examined for each level of porosity to evaluate variations induced by the statistical nature of the microstructure. In all cases, variations of less than $2 \%$ were observed. The effect of the finite model size was investigated by gradually increasing the linear dimensions of the model by up to a factor of ten, while keeping pore size level of porosity fixed. The error induced by the finite mesh size was 
evaluated by refining the mesh size by a factor of ten. Convergent results were obtained with simulation errors smaller than the statistical variations in the microstructure as long as the edge length of the model was at least 50 times larger and the mesh seed size 10 times smaller than the characteristic pore radius.

\section{$\underline{\text { Results and discussion }}$}

Figure 3(a) shows representative FTIR absorption spectra for the OSG films. Figure 3(b) provides a survey of the bonds that occur in the OSG coatings as a function of film porosity. The bond densities in the figure were calculated from the FTIR absorption spectra of the coatings using inverse infrared cross-sections as discussed in Reference [17] and represent bond densities for the OSG matrix, rather than averages over the entire film volume. The densities of both the siloxane bonds and the organic component in the matrix of the porous films increase with increasing porogen. Evidently, the intensity of the UV radiation is less attenuated by the more porous films, causing a more effective densification of the matrix after the porogen has been removed from the films. The enhanced organic component may be attributed to porogen residuals in the films. Lin et al. [15] have demonstrated that the mechanical properties scale with the density of networking bonds in OSG. They developed an empirical parameter, the weighted networking bond density or $W N B D$, that shows a strong correlation with both the OSG stiffness and fracture energy over a wide range of deposition parameters. The $W N B D$ is the total number density of the two main networking bonds found in OSG, i.e., the siloxane bond and the methylene bond, where the density of methylene bonds is normalized for its lower bond strength: one Si-O bond counts for one networking bond, whereas one $\mathrm{Si}-\mathrm{CH}_{2}$ bond counts for only 0.56 networking bond. The contribution of 
the methylene bonds is reduced as they add less to the overall stiffness and fracture energy of the material because of their lower bond energy. The $W N B D$ parameter is readily calculated from the FTIR spectra of the films and is listed in Table 1 for all OSG specimens.

Table 1 also lists the plane-strain moduli and the fracture energies of the OSG films. As expected, both stiffness and cohesive energy decrease with increasing porosity, but it is evident that the stiffness decreases much faster than the cohesive energy. The change in both parameters can be attributed to two factors: 1) A change in the stiffness or fracture energy of the OSG matrix as a result of the increased porogen loading during the deposition process and the corresponding enhanced densification during UV irradiation, and 2) a decrease in these properties as a direct result of the increased porosity. These two contributions will now be discussed in more detail.

The plane-strain elastic moduli of the OSG films can be written as

$$
M=M_{\text {Matrix }}\left(W N B D_{\text {Matrix }}\right) f(p)
$$

where $M_{\text {Matrix }}\left(W N B D_{\text {Matrix }}\right)$ is the plane-strain modulus of the matrix and $f(p)$ is a general function that expresses the effect of the porosity $p$ and that should be the same for all materials with a similar pore microstructure. As the porogen loading is increased during the deposition process, the OSG matrix structure is modified as reflected by the dependence of its modulus on the network bond density of the matrix, $W N B D_{\text {Matrix }}$. Lin et al. [18] have demonstrated that there exists a linear correlation between the WNBD of an OSG material and its elastic modulus. This correlation is shown in Fig. 4 for a number of dense OSG films deposited under a wide range of process conditions (redrawn from reference [18]). The modulus of the dense matrix can then be written as 


$$
M_{\text {Matrix }}=\beta+\alpha W N B D_{\text {Matrix }},
$$

where $\alpha$ and $\beta$ are curve fit parameters that can be determined from a linear least squares fit to the data in Fig. 4. The network bond density of the matrix, $W N B D_{\text {Matrix }}$, is related to the overall bond density of the OSG film, $W N B D_{\text {Film }}$, through

$$
W N B D_{\text {Matrix }}=W N B D_{\text {Film }} /(1-p) \text {. }
$$

The values of $W N B D_{\text {Film }}$ as measured by FTIR are listed in Table 1 for all OSG samples. Combining Eqs. (4), (5), and (6) leads to the following expression for the effect of the porosity on the stiffness of the OSG films

$$
f(p)=\frac{(1-p) M}{\beta(1-p)+\alpha W N B D_{F i l m}} .
$$

Figure 5(a) shows the experimental values of $f(p)$ for the OSG films. The function $f(p)$ represents the intrinsic effect of porosity on the stiffness of the OSG films and can be compared with theoretical models and simulations to get insight in the pore microstructure of the films. The experimental results in Fig. 5(a) are compared with finite element simulations of the non-overlapping spherical pore model, as well as with several models published in the literature, including the random overlapping spherical pore model (FEM) [11], the random solid sphere model (FEM) [11], and the HashinShtrikman upper bound [4]. Implicit in this comparison is the assumption that Young's modulus and the plane-strain modulus have the same porosity dependence. The experimental values are all smaller than the corresponding Hashin-Shtrikman bounds. At low OSG film porosity, the experimental result is in agreement with the models representing microstructures that consist of randomly distributed spherical pores, 
regardless of whether the pores overlap or not. When the OSG porosity increases further, however, the experimental results track the solid sphere model, which mimics a microstructure of sintered ceramic particles. This observation suggests that the microstructure of the OSG coatings evolves with increasing porosity - going from a structure with isolated pores for films with low porogen loading to a structure with greatly interconnected pores for films with higher porogen contents. This evolution has indeed been observed in similar dielectrics using positronium annihilation lifetime spectroscopy [11]. It is further evident from the figure that the stiffness of a porous solid depends sensitively on its precise microstructure. Isolated pores, for example, result in a stiffer material than do overlapping pores, which in turn result in a significantly stiffer material than sintered spheres. Depending on the overall porosity, the difference between the microstructures can be as large as a factor of two. This result implies that it may be possible to significantly increase the stiffness of an OSG film with a given porosity by optimizing its pore microstructure, for instance, through use of a porogen that minimizes clustering of the porogen phase in as-deposited coatings.

The non-overlapping spherical pore microstructure also offers a good model to explore the effect of pore size distribution on the overall stiffness of the OSG. Figure 5(b) shows FEM results for the normalized modulus as a function of porosity for a model where all pores are of the same size ( $1 \%$ of the edge length of the model) and for a model where the pore size is uniformly distributed between 0.5 and $1.5 \%$ of the linear dimension of the model. There is no significant difference between the two pore microstructures in the range of 0 to $40 \%$ porosity. Consequently, it seems advisable to 
use porogens that create a distribution of pore sizes given that it is easier to avoid interconnected pores if a range of pore sizes is available.

Finally it should be pointed out that agreement of the experimental results with the solid sphere model does not necessarily imply that this model also best represents the microstructure of the OSG films. In all the models shown in Fig. 5(a) only spherical pores or spheres were considered. Evidently, the stiffness of a porous material also depends on the shape of the pores [4]. Even though we expect the pores in OSG films with relatively low levels of porosity to be more or less equi-axed, no detailed information on pore shape is available. An in-depth experimental investigation of pore shape effects would require other experimental techniques and is beyond the scope of this paper.

Figure 6 shows the fracture energy of a series of both dense and porous OSG coatings as a function of the weighted network bond density, $W N B D$, of the OSG. It is evident from the figure that the fracture energy of the dense OSG scales linearly with $W N B D$. This linear relationship is not surprising because fracture of brittle materials such as OSG is a bond breaking process and the energy dissipated during fracture should scale with the number of broken networking bonds and with the energy of the bonds. This observation is used to correct for any changes in the matrix structure of the porous OSG film as a result of different processing conditions. Similar to Eq. (4), we write the fracture energy, $G$, of the OSG coatings in terms of the fracture energy of the matrix, $G_{\text {Matrix }}$, and a function $g(p)$ of the porosity:

$$
G=G_{\text {Matrix }}\left(W N B D_{\text {Matrix }}\right) g(p) .
$$


The effect of process conditions on the fracture energy of the OSG matrix is captured by a linear function of the weighted network bond density of the matrix,

$$
G_{\text {Matrix }}=\gamma+\chi W N B D_{\text {Matrix }}
$$

where $\gamma$ and $\chi$ are curve fit parameters that can be determined from a linear least squares fit to the data in Fig. 6. As before, the network bond density of the matrix, $W N B D_{\text {Matrix }}$, is related to the overall bond density of the OSG film, $W N B D_{\text {Film }}$, through Eq. (6). The effect of porosity on the fracture energy of the OSG coatings is then given by

$$
g(p)=\frac{(1-p) G}{\gamma(1-p)+\chi W N B D_{\text {Film }}}
$$

Experimental values of the function $g(p)$ are plotted in Fig. 7(a). The results in this figure demonstrate that the fracture energy of the OSG coatings decreases linearly with increasing porosity for porosity values up to $45 \%$. This linear scaling is consistent with a planar through-pore fracture mechanism as illustrated in Fig. 7(b). In such a mechanism, the solid area that must be fractured changes linearly with film porosity; effects associated with pore shape, size, or distribution are screened out for a random pore microstructure. This linear relationship between fracture energy and film porosity stands in contrast with the power-law behavior (exponent $\sim 2$ ) measured by Guyer et al. [13] for MSSQ coatings using the four-point bend flexure technique. This nonlinear behavior was attributed by these authors to several factors including the non-uniformity of the MSSQ density through the coating thickness and contributions from frictional contact between the crack faces. These factors are important under the mixed-loading conditions of the four-point flexure test, but should have minimal effect under the mode I loading conditions of the DCB technique. Our results indicate that in the absence of 
these complicating factors the cohesive energy does scale linearly with the density of the atomic bonds on the fracture plane.

Furthermore, no threshold porosity is observed, below which the fracture energy is independent of porosity, as reported for crack propagation in PMMA with cylindrical pores or in porous alumina samples [13]. This effect has been attributed to crack front bowing between pores, which causes the driving force to propagate the crack to increase beyond the force that is required to drive the crack in a pore-free material. If indeed crack front bowing takes place in porous OSG, no significant effect is found on the measured fracture energy. Instead, it is likely that the pores are too small to have any pinning effect on the propagating crack and the OSG fracture energy decreases monotonically with increasing porosity in the range investigated.

To examine the hypothesis of the fracture mechanism in more detail, a systematic AFM study was carried out with the aim of characterizing the morphology of the OSG fracture surfaces as a function of porosity. Both the scan area and step size were varied to evaluate the roughness of the crack faces at different length scales. Figures $8(\mathrm{a})-8(\mathrm{c})$ show typical AFM scans for OSG films with different levels of porosity. All fracture surfaces are very smooth with an RMS roughness of a few nanometers at most, as summarized in Fig. 8(d). Even so, the surface morphology has a somewhat granular appearance with a characteristic wavelength on the order of a few tens of nanometers that decreases with increasing porosity. This granular morphology has also been observed for fully dense OSG films [24] and may be related to a microstructural inhomogeneity in the OSG at this length scale that causes the crack to meander around its average position seeking out the path of least resistance. There is a very small but systematic increase in roughness with increasing porosity. The resulting 
increase in total fracture area, however, is too insignificant to have any effect on the experimentally measured fracture energy - the effect of the OSG porosity on the morphology of the fracture surfaces is too subtle to be reflected in the fracture energy of the coatings.

\section{$\underline{\text { Conclusions }}$}

We have investigated the effect of porogen content on the stiffness and cohesive fracture energy of PECVD OSG coatings. The porogen content affects both the network structure and the porosity of the dielectric and we have evaluated the contributions of both effects to the stiffness and fracture energy of the films. The stiffness results show that the OSG microstructure evolves with increasing porosity, from a structure with isolated pores for films with low porogen loading to one with heavily interconnected pores at higher porogen contents. When compared with FEM simulations, the results further suggest that the OSG stiffness can be significantly enhanced if the pore microstructure is optimized to reduce pore connectivity. The fracture energy of the films decreases linearly with increasing porosity, consistent with a simple planar through-pore fracture mechanism.

\section{Acknowledgments}

This research was supported by the Semiconductor Research Corporation (Task ID: 1292.010). The OSG film stacks were provided by Texas Instruments Incorporated. The help of Nancy Ota with nanoindentation measurements is also gratefully acknowledged. 


\section{REFERENCES}

1. Mark L. O'Neill, Raymond N. Vrtis, Brian K. Peterson, Mary K. Haas, Scott J. Weigel, Dingjun Wu, M.D. Bitner, and E.J. Karwacki. Impact of Pore Size and Morphology of Porous Organosilicate Glasses on Integrated Circuit Manufacturing. in Materi. Res. Soc. Spring, Symposium F. 2006.914. Ting Y. Tsui, Young-Chang Joo, Lynne Michaelson, Michael Lane, and A.A. Volinsky. p 0914-F01-02.

2. Maex, K., M.R. Baklanov, D. Shamiryan, F. Iacopi, S.H. Brongersma, and Z.S. Yanovitskaya, Low Dielectric Constant Materials for Microelectronics. Journal of Applied Physics, 2003. 93(11): p. 8793-8841.

3. Morgen, M., E.T. Ryan, J.H. Zhao, C. Hu, T.H. Cho, and P.S. Ho, Low Dielectric Constant Materials for ULSI Interconnects. Annual Review of Materials Science, 2000. 30: p. 645-680.

4. Sia Nemat-Nasser and M. Hori., Micromechanics : Overall Properties of Heterogeneous Materials 2nd rev. ed. ed. 1999, Amsterdam New York: Elsevier.

5. Roscoe, R., The Viscosity of Suspensions of Rigid Spheres. British Journal of Applied Physics 1952. 3(8): p. 267-269.

6. Christensen, R.M. and K.H. Lo, Solutions for Effective Shear Properties in 3 Phase Sphere and Cylinder Models. Journal of the Mechanics and Physics of Solids, 1979. 27(4): p. 315-330.

7. Benveniste, Y., A New Approach to the Application of Mori-Tanaka Theory in Composite-Materials. Mechanics of Materials, 1987. 6(2): p. 147-157.

8. Eshelby, J.D., The Determination of the Elastic Field of an Ellipsoidal Inclusion, and Related Problems. Proceedings of the Royal Society of London Series AMathematical and Physical Sciences 1957. 241(1226): p. 376-396.

9. Rice, R.W., Evaluation and Extension of Physical Property-Porosity Models Based on Minimum Solid Area Journal of Materials Science 1996. 31(1): p. 102-118.

10. Torquato, S., Effective Stiffness Tensor of Composite Media .1. Exact Series Expansions Journal of the Mechanics and Physics of Solids 1997. 45(9): p. 1421-1448.

11. Roberts, A.P. and E.J. Garboczi, Elastic Properties of Model Porous Ceramics. Journal of the American Ceramic Society, 2000. 83(12): p. 3041-3048.

12. Roberts, A.P. and E.J. Garboczi, Computation of the Linear Elastic Properties of Random Porous Materials with a Wide Variety of Microstructure.

Proceedings of the Royal Society of London Series a-Mathematical Physical and Engineering Sciences, 2002. 458(2021): p. 1033-1054.

13. Vandeperre, L.J., J. Wang, and W.J. Clegg, Effects of Porosity on the Measured Fracture Energy of Brittle Materials. Philosophical Magazine, 2004. 84(34): p. 3689-3704.

14. Guyer, E.P., M. Patz, and R.H. Dauskardt, Fracture of Nanoporous Methyl Silsesquioxane Thin-film Glasses. Journal of Materials Research, 2006. 21(4): p. 882-894. 
15. Youbo Lin, Yong Xiang, Ting Y. Tsui, and J.J. Vlassak, PECVD lowpermittivity organosilicate glass coatings: Adhesion, fracture and mechanical properties. Acta Materialia, 2008. 56(17): p. 4932-4943.

16. Baklanov, M.R., K.P. Mogilnikov, V.G. Polovinkin, and F.N. Dultsev, Determination of Pore Size Distribution in Thin Films by Ellipsometric Porosimetry. Journal of Vacuum Science \& Technology B, 2000. 18(3): p. 1385-1391.

17. Lin, Y.B., T.Y. Tsui, and J.J. Vlassak, Octamethylcyclotetrasiloxane-based, Low-permittivity Organosilicate Coatings - Composition, Structure, and Polarizability. Journal of the Electrochemical Society, 2006. 153(7): p. F144F152.

18. Lin, Y.B., Y. Xiang, T.Y. Tsui, and J.J. Vlassak, PECVD Low-Permittivity Organosilicate Glass Coatings: Adhesion, Fracture and Mechanical Properties. Acta Materialia, 2008. submitted.

19. Oliver, W.C. and G.M. Pharr, An Improved Technique for Determining Hardness and Elastic-Modulus Using Load and Displacement Sensing Indentation Experiments. Journal of Materials Research, 1992. 7(6): p. 15641583.

20. Chen, X. and J.J. Vlassak, Numerical Study on the Measurement of Thin Film Mechanical Properties by Means of Nanoindentation. Journal of Materials Research, 2001. 16(10): p. 2974-2982.

21. Kanninen, M.F., Augmented Double Cantilever Beam Model for Studying Crack-Propagation and Arrest. International Journal of Fracture, 1973. 9(1): p. 83-92.

22. Li, S., J. Wang, and M.D. Thouless, The effects of shear on delamination in layered materials. Journal of the Mechanics and Physics of Solids, 2004. 52(1): p. 193-214.

23. Roberts, A.P. and E.J. Garboczi, Elastic Properties of Model Random Threedimensional Open-cell Solids. Journal of the Mechanics and Physics of Solids, 2002. 50(1): p. 33-55.

24. Vlassak, J.J., Y. Lin, and T.Y. Tsui, Fracture of Organosilicate Glass Thin Films: Environmental Effects. Materials Science and Engineering a-Structural Materials Properties Microstructure and Processing, 2005. 391(1-2): p. 159-174. 


\section{TABLES AND FIGURES}

\begin{tabular}{ccccc}
\hline $\begin{array}{c}\text { Porosity } \\
\%\end{array}$ & $\mathbf{k} @ \mathbf{a} \mathbf{M H z}$ & $\begin{array}{c}\text { Overall } \mathbf{W N B D} \\
\left(\mathbf{1 0} \mathbf{1}^{\mathbf{2}} \mathbf{)}\right.\end{array}$ & $\begin{array}{c}\text { Plane-strain modulus } \\
(\mathbf{G P a})\end{array}$ & $\begin{array}{c}\text { Cohesive fracture } \\
\text { energy }\left(\mathbf{J} / \mathbf{m}^{2}\right)\end{array}$ \\
\hline 7.0 & 3.01 & 1.37 & $18.6 \pm 0.5$ & $3.73 \pm 0.40$ \\
7.4 & 2.98 & 1.35 & $18.4 \pm 0.9$ & $3.50 \pm 0.39$ \\
14.8 & 2.76 & 1.22 & $-\mathrm{NA}-$ & $3.30 \pm 0.20$ \\
18.9 & 2.62 & 1.20 & $8.0 \pm 0.4$ & $3.03 \pm 0.34$ \\
20.1 & 2.60 & 1.14 & $7.4 \pm 0.4$ & $2.78 \pm 0.40$ \\
27.2 & 2.43 & 1.05 & $4.5 \pm 0.2$ & $2.68 \pm 0.17$ \\
44.5 & 2.30 & 1.00 & $3.5 \pm 0.1$ & $2.40 \pm 0.10$ \\
\hline
\end{tabular}

Table I. $\quad$ Porosity, overall energy-weighted network bond density $W N B D$, plane-strain modulus, and cohesive energy of the OSG specimens. 


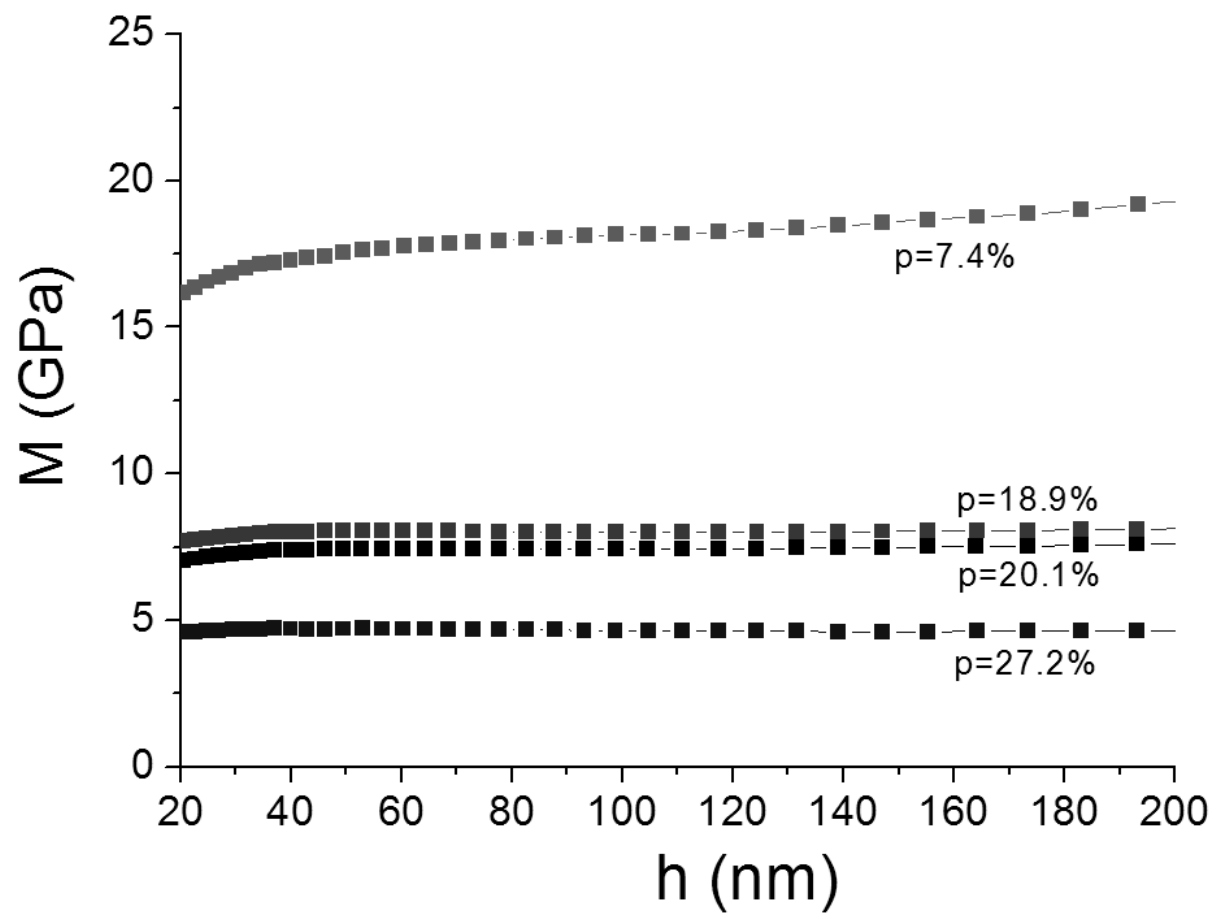

Figure 1. Plane-strain elastic modulus as a function of indentation depth for a representative subset of the porous OSG films. 


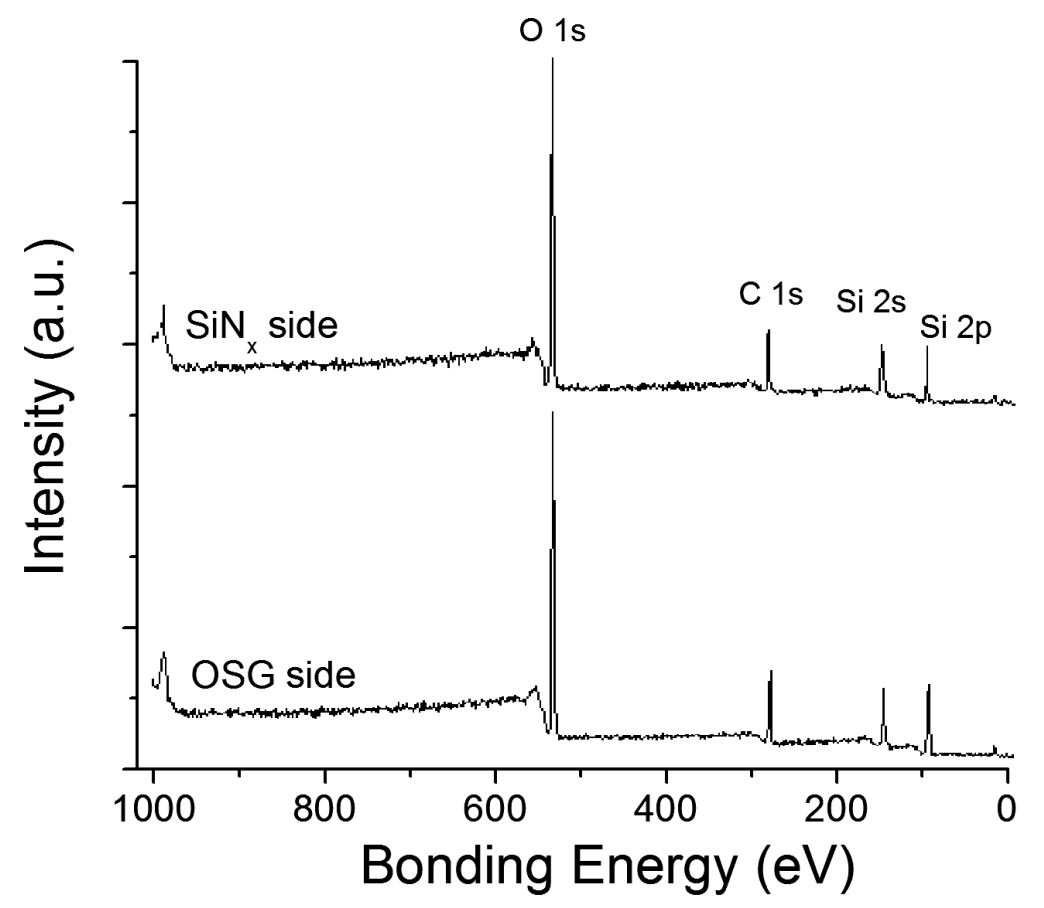

Figure 2. Typical XPS spectra of the fracture surfaces of an OSG film with a porosity of $14.8 \%$ after the DCB test. The near-identical spectra of both surfaces indicate that fracture propagates within the OSG films. 


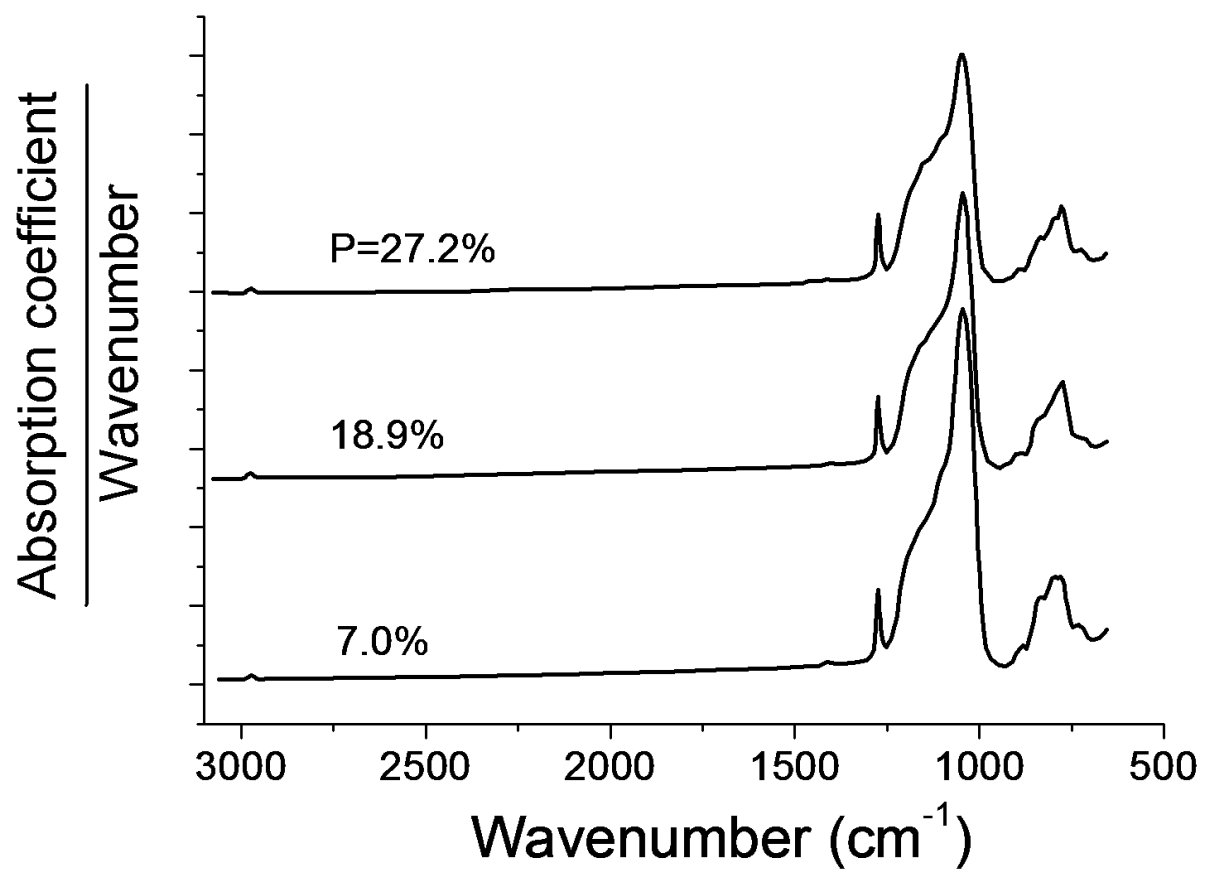

(a)

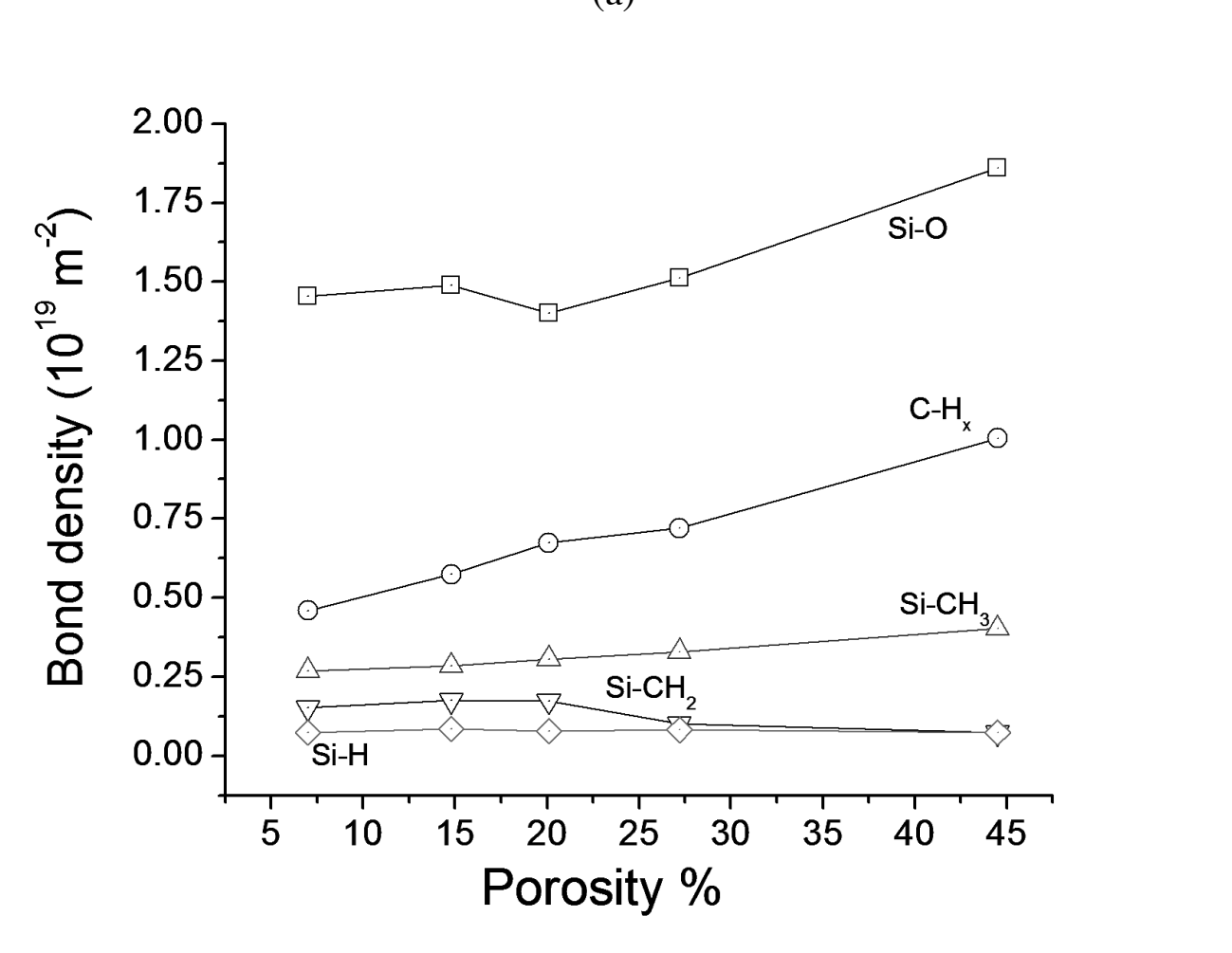

(b)

Figure 3. (a) Typical FTIR absorption spectra of the OSG films; spectra are offset for comparison purpose. (b) Survey of the bond densities in porous OSG films with different levels of porosity, calculated from the FTIR spectra using the inverse infrared cross sections from reference [17]. 


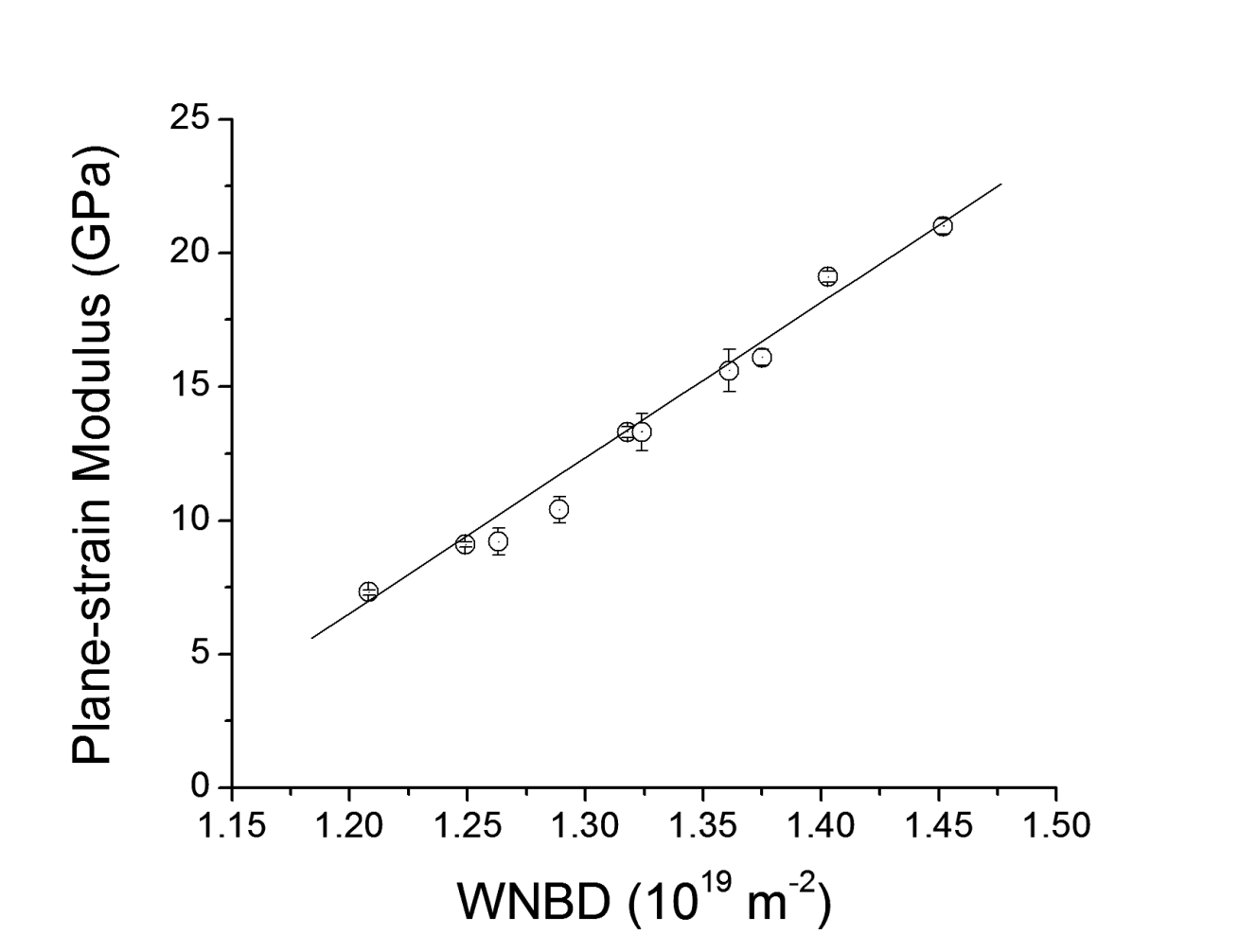

Figure 4. Plane-strain modulus of a series of dense OSG films as a function of WNBD; data taken from [13]. 


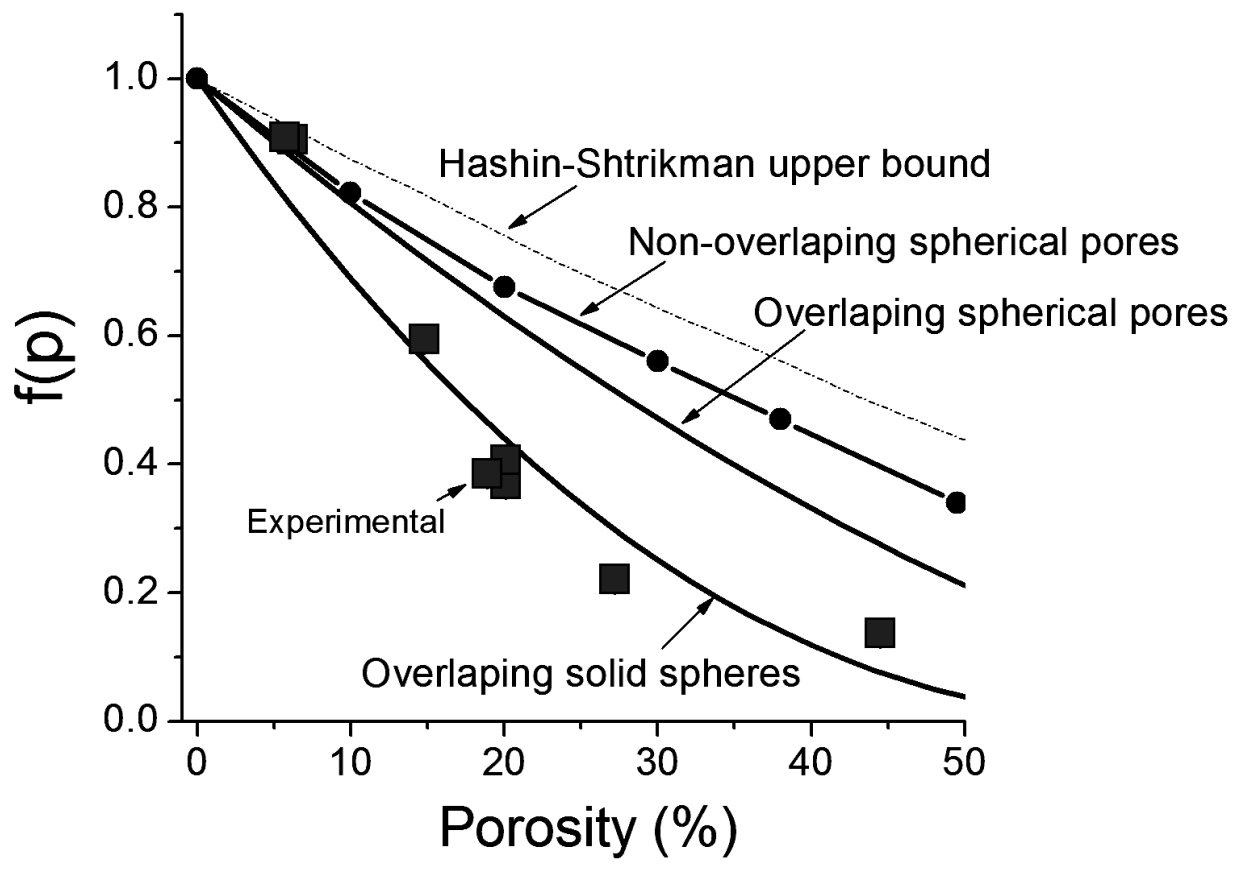

(a)

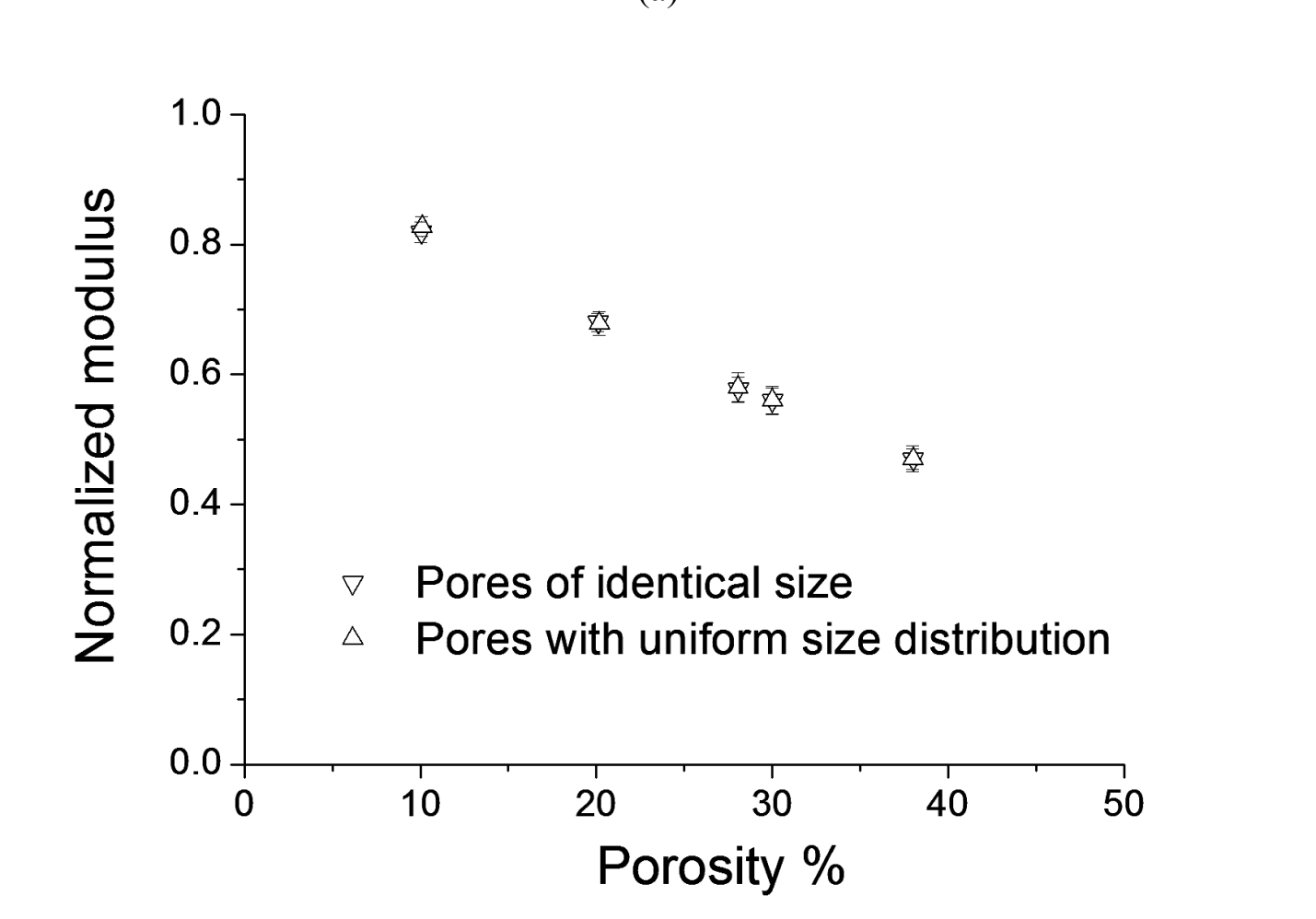

(b)

Figure 5. (a) Experimental values of the function $f(p)$, compared with finite element simulations of various pore microstructures. (b) Effect of the pore size distribution on the effective modulus of a porous material with non-overlapping pores. There is no significant difference between materials with monodispersive or multidispersive pore distributions. 


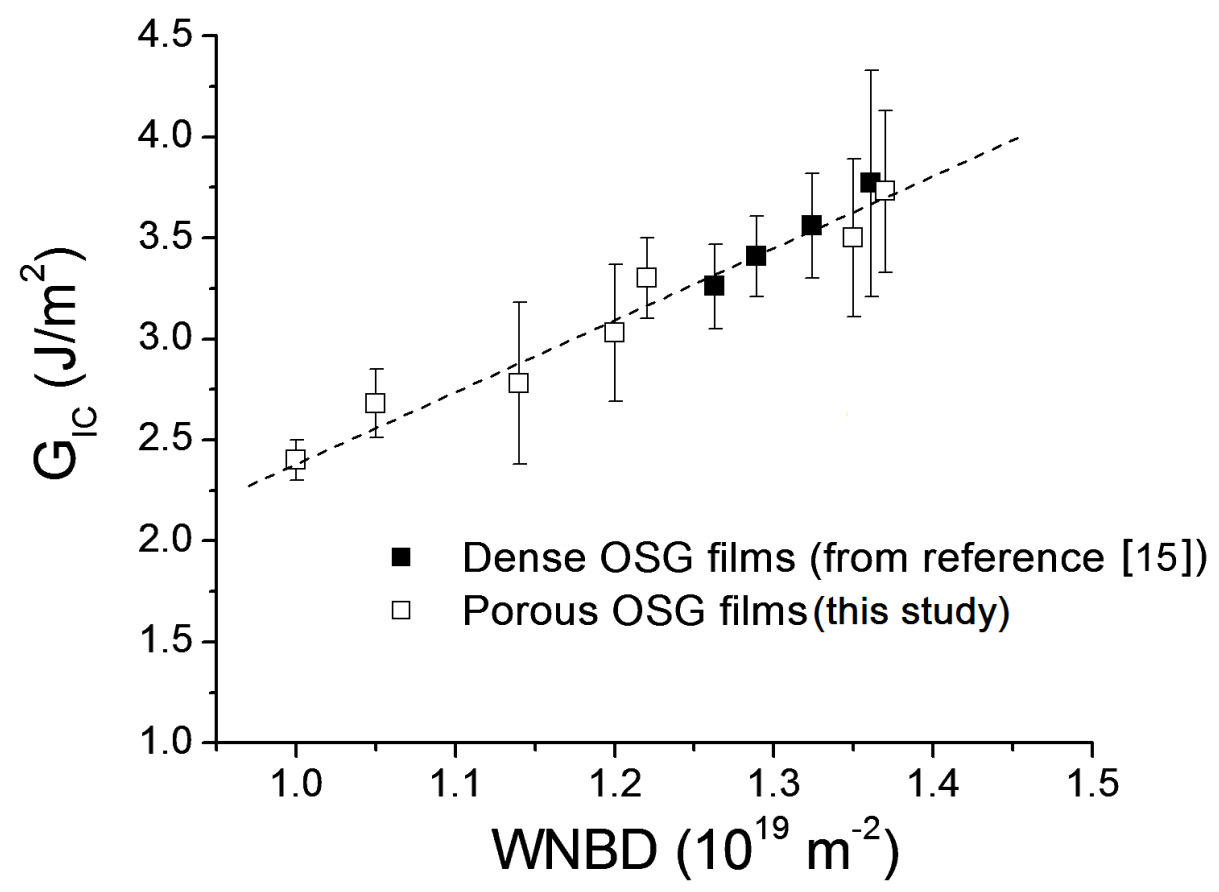

Figure 6. $\quad$ Fracture energy of OSG as a function of overall $W N B D$. Data for both dense and porous OSG films are shown. 

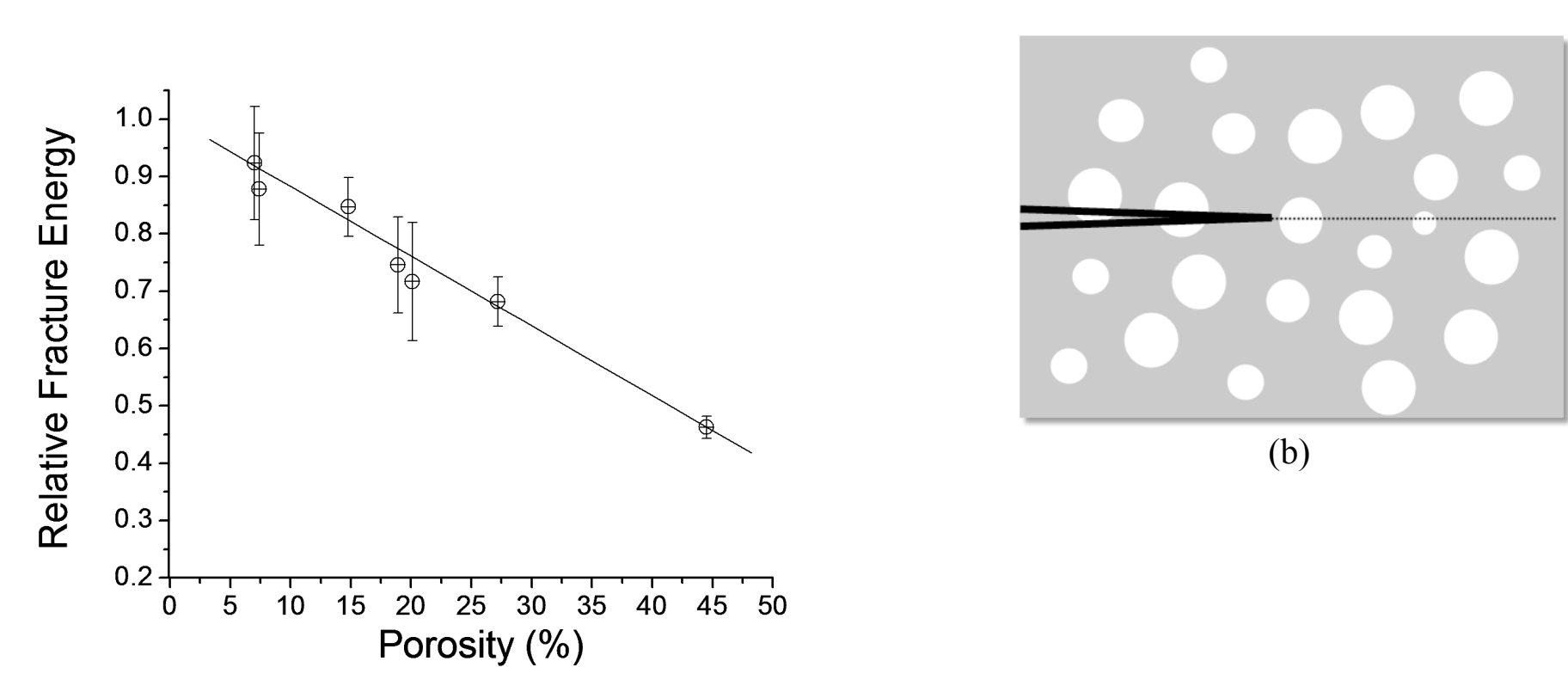

(b)

(a)

Figure 7. (a) Effect of porosity on the fracture energy of OSG as expressed by $g(p)$. (b) The number of bonds that need to be fractured is proportional to (1-p) in the planar throughpore fracture mechanism. 


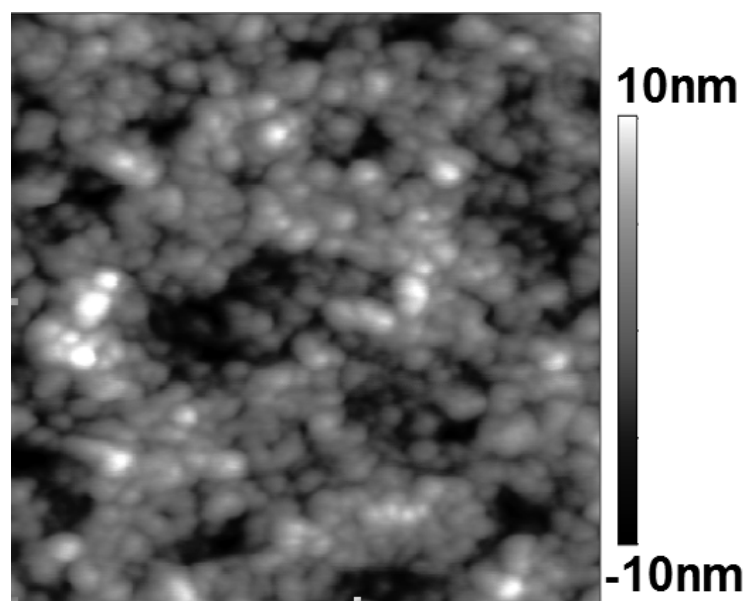

(a)

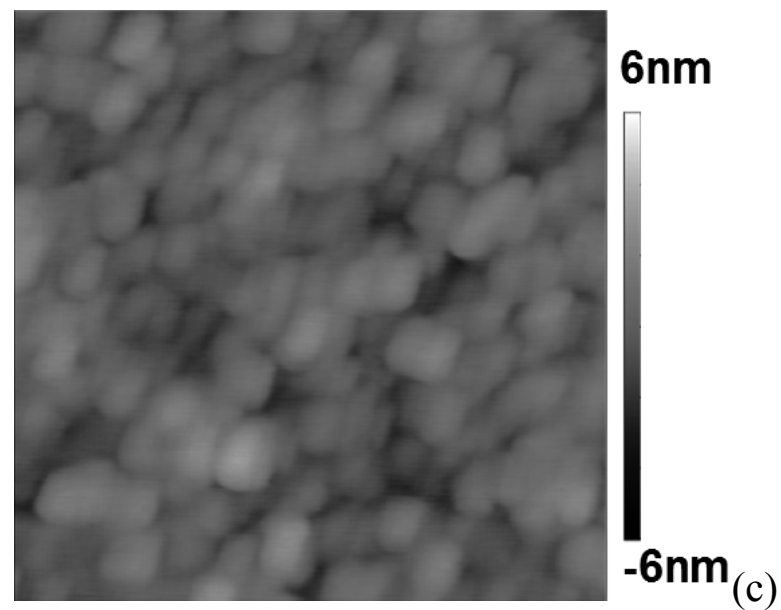

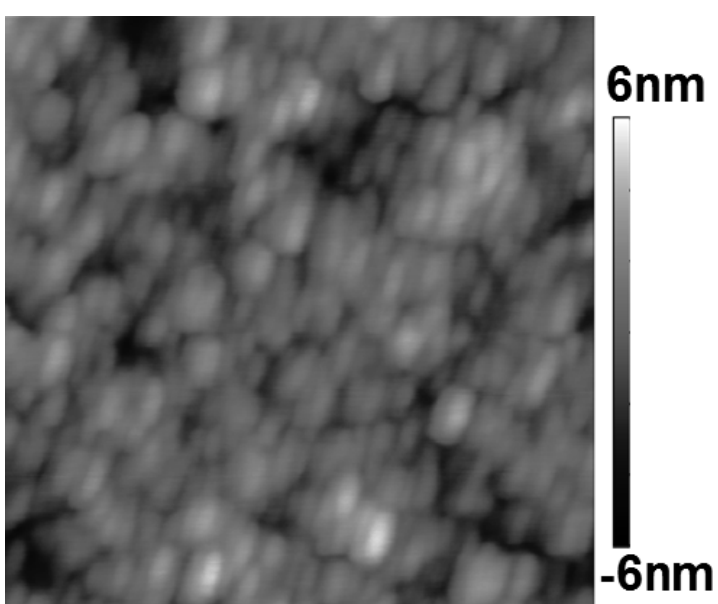

(b)

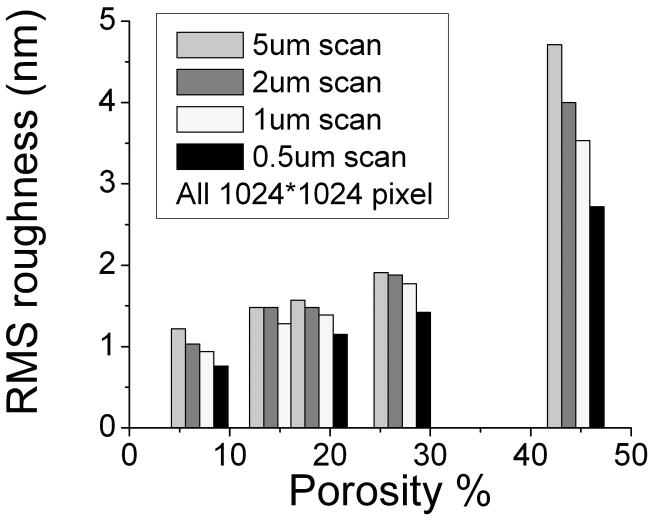

(d)

Figure 8. AFM micrographs of fracture surfaces $\left(1 \times 1 \mu \mathrm{m}^{2}\right)$ for films with porosity levels of (a) $p=44.5 \%$, (b) $p=18.9 \%$, (c) $p=7 \%$. (d) RMS roughness as a function of film porosity and scan size. 